\title{
Approach of Car License Plate Location based on Improved Sobel Operator with the Combination of Mathematical Morphology
}

\author{
YanlingCui ${ }^{1}$ and Chengjun Yang ${ }^{2 *}$ \\ ${ }^{1}$ Institute of Computer Science and Information Engineering, HarbinNormalUniversity, \\ Harbin 150080, China \\ ${ }^{2}$ College of Forestry, NortheastForestryUniversity, Harbin 150040, China \\ ${ }^{1}$ cuiyanlinghsd@163.com, ${ }^{2}$ nxyycj@sina.cn
}

\begin{abstract}
Plate license locating is the key process in vehicle license plate recognition for automated transport system. It will affect the accuracy of recognition directly. License plate area contains rich edge and texture information. In this paper, improved Sobel operator is used to detecting the edge of the car image. This paper presents a new approach of extracting car license plate from car images based on morphology. Based on the information of width and height of car license plate, and space between characters of the car license plate, we design a new structural elements for morphological operations used to the edge image of car license plate, which can obtain several candidate areas. Finally, the criterions basing on the ratio of plate area, ratio of height to width and projections are used in comprehensive analysis, and the real license plate area can be located accurately. The improved Sobel operator method is helpful to reveal distinct characteristics for some edge features, efficiently with high speed processing. Compared with the classical edge detection operators edge detection algorithm, the improved algorithm is more advantage, the quality and speed of the picture edge extraction significantly improved. Experimental results show that this method is simple, accurate.It can satisfy the real time need of license location demand, and can make the preparation for the following character identification.
\end{abstract}

Keywords:Mathematical Morphology; Structure element; Sobel edge detection; License Plate Location

\section{Introduction}

Nowadays license plate recognition (LPR) becomes a key technique to many automated transport systems such as road traffic monitoring, automatic payment of tolls on highways or bridges and parking lots access control. A LPR system is mainly composed of three processing modules: that is, license plate location, the character segmentation, and character recognition. Among them, the license plate location is considered as the most crucial stage in the whole LPR systemand it has received considerable attention[1]. The most important and basic one is to correctly extract the position of the vehicle license, which directly affects system's overall accuracy. In developed countries, LPR system has been successfully applied in the actual transportation system, but its development is relatively slow in china, staying in laboratory stage. There are three reasons for this situation; firstly, there are many styles in Chinese car license, the standard is inconsistent, and the car license position is also various; Secondly, weather, lighting and motion and other factors have a great influence on the license plate image. Thirdly, someof license plates wear badly, so that the information is vague. The

\footnotetext{
* Corresponding Author
} 
above factors will have a greateffect on the accuracy of car license localization, thus it needs to explore reasonable and effective localization algorithm to overcome the influence of environment and external factors. There are many kinds of car license localization's methods at present time. They are in the edge detection, the projection, gray feature method, transform method, neural network and so on. These methods have their own advantages, but there are some shortages, such as computational is intensive, positioning accuracy defects is not high [2]. In recent years, the morphology image processing, as the special image processing discipline, has become a major research field, there are many research papers at home and abroad that it is applied to extract the text[3], but used to license plate extraction needs further exploration. In this paper, improved Sobel operator is used to detecting the edge of the car image, combining with mathematical morphology. Based on the information of width and height of car license plate and space between characters on it, designs a new structural element for morphological operations, which can obtain several candidate areas. Finally, the criterions basing on the plate area ratio, height to width ratio and projections are used in comprehensive analysis, and the real license plate area can be located accurately.

\section{Gray-scale Image}

Chinese car license's bottom color and the character color mainly have 4 kinds, they are, the white character on the blue background, the black character on the yellow background, the white character on the black background and the black character on the white background. Because the color is not unified, this article uses the gray image to carry on processing [4]. The gray processing method mainly has three kinds: maximum value method, mean value method and weighted average method. In this paper, the weighted average method is applied, car license image's original color R, G, B are computed according to the formula 2-1 to get a new gray value, the original image is converted to gray image.

$$
f(x, y)=0.299 \times R+0.587 \times G+0.114 \times B
$$
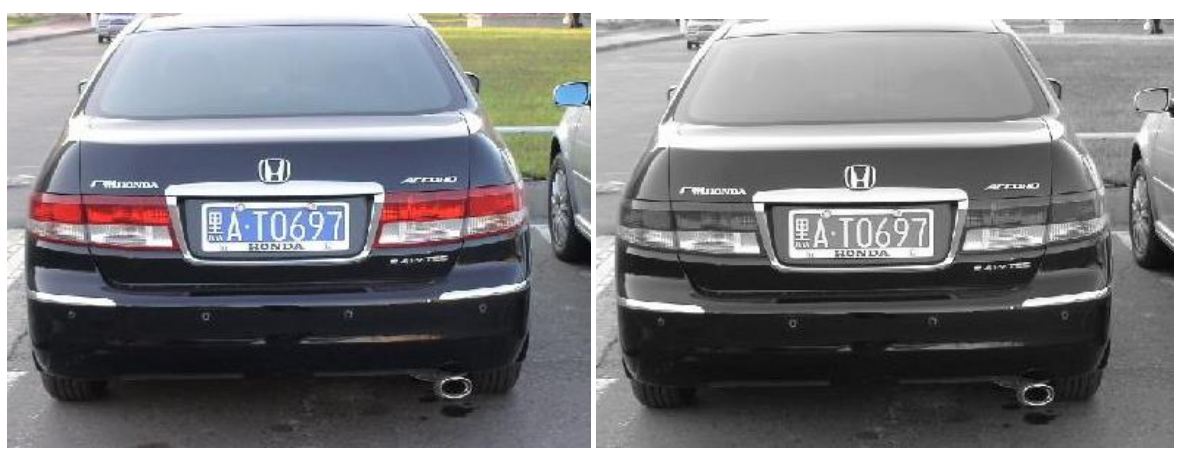

Figure 1. Original Car Image; Gray Car Image

\section{Edge Detection}

Because these plates have the common characteristics, namely gray value of the background and the character and the border have great contrast aftergray, so we firstly carry on edge detection, and by using the edge detection operator on the pretreatment to highlight edge texture. Commonly edge detection operators have Roberts, Sobel, Prewitt and Krisch.Prewitt edge detection operator is the advantage of simple calculation, to noise strong inhibition, but the drawback is that the result has the fuzzy edge vague, with many 
intermittent points, and the vertical edge detection is not strong. Roberts is a simple edge detector that is very limited in functionality [5].Through the observed image of the car: license plate contains the characters, the vertical texture is dense and rich, but the automobile body image is actually the opposite. That is in the license plate the vertical edge texture must be much richer than the horizontal edge texture[6].Therefore, firstly we carry on image edge sharpening, which are used to enhance vertical edge, eliminate most of the non-license plate area. But the use of above operators on the vertical edge in the extraction is not strong, the effect is shown in Figure 2, for this reason we use Sobel operator as edge detection.
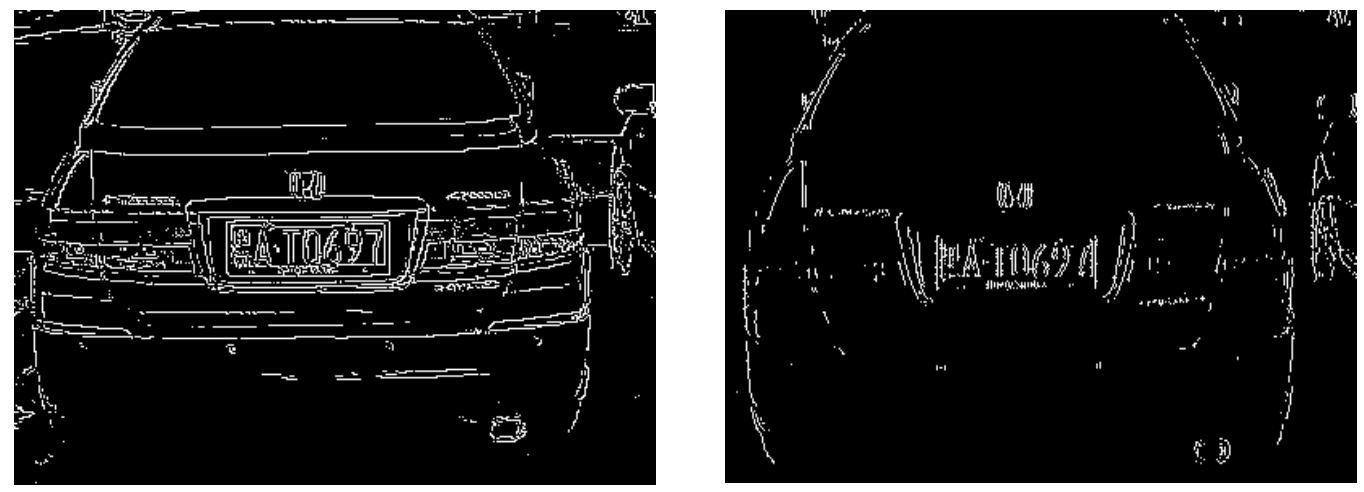

\section{Figure 2. Edge Detection with Roberts Operator;Vertical Edge Detection with Prewitt Operator}

There are only horizontal and vertical directions templates in traditional Sobel operator, therefore response is biggest to the vertical and the horizontal edge, which is good to these images of the gradation and the many noise.Calculation principle is the use of two convolution kernels with each point in the plate image making convolution, and the maximum in the convolved results is used as the output of this point, then we make output resultto binaryzation, and form the binary image finally.Through analyzing, we can know that texture edge detection is only sensitive to the vertical. In order to further enhance the vertical edges, so we make the improvement to the traditional Sobel operator, uses three templates: vertical directions, 45 degrees directions, the -45 degrees directions to detect the plate image. The templates are shown below.

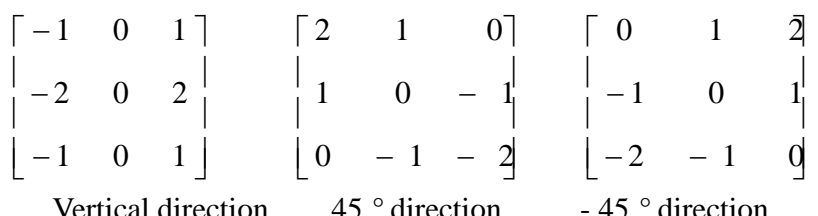

We are carrying on the point-by-point computation using the above three templates,take the maximum value as the new gray level of this point, and the maximum value that corresponds to the direction of the template is edge direction of this pixel. Test results are shown in Figure 3: 

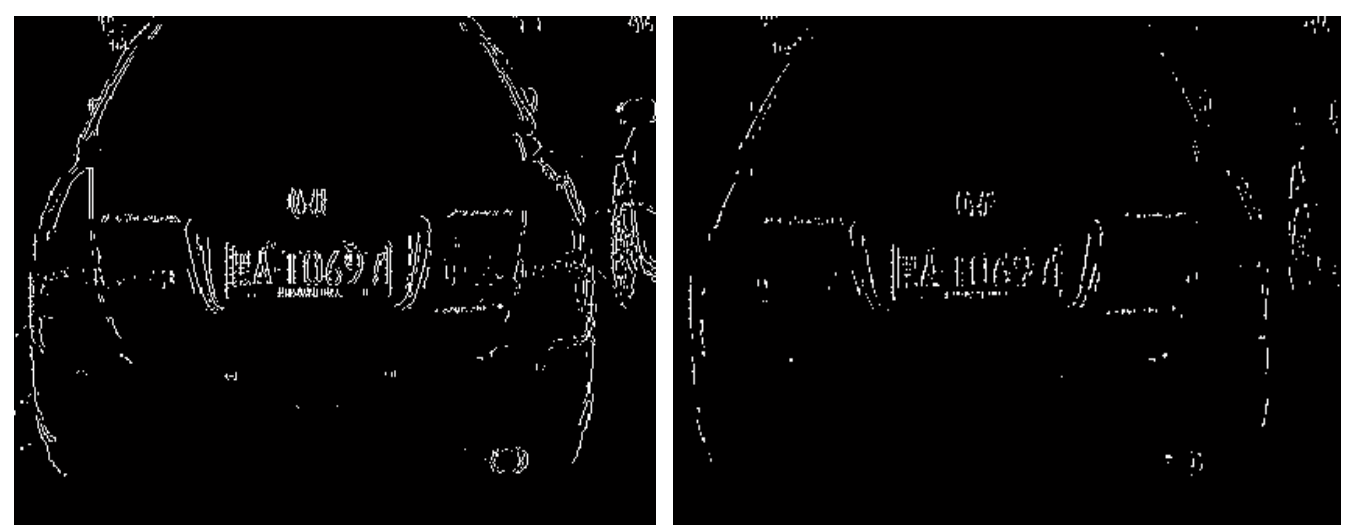

Figure 3. Vertical Edge Detection with Traditional Sobel Operator; Vertical Edge
Detection with Improved Sobel Operator

It can be seen from two figures: through the improved Sobeloperation, we can get license region to be located and other regions border.Improved Sobel is obviously superior to the traditional Sobel operator in complex the edge feature.

\section{Mathematical Morphology Location}

Mathematical morphology is developed on the basis of the set theory[7]. It is widely used in analyzing and processing of image shape and structure: image segmentation, feature extraction, edge detection, image filtering, image enhancement and restoration etc. It has four basic operations for erosion, dilation, closed and open. Erosion operation is used to eliminate the object boundary point. It is a process of shrinking to internal boundary, and object less than structural elements can be removed. On the contrary, the dilation is carries on the expansion to the image boundary point, conjoint background points are merged, so dilation is the boundary to the exterior expansion process. Opening operation is used to filter the details thatare less than the structural element; closing operation is to fill the details of structural elements [8]. Their expressionsare defined as:

Dilation operation:

$$
A \oplus B=\{c: c \in Z \mid c=a+b, a \in A, b \in B\}=\bigcup_{a \in A} B[a]=\bigcup_{b \in B} X[b]
$$

Erosion operation:

$$
A \Theta B=\{c: c \in Z \mid c+b \in A, b \in B\}=\bigcap_{b \in B} A[b]
$$

Opening operation:

$$
A \circ B=(A \Theta B) \oplus B
$$

Closing operation:

$$
A \bullet B=(A \oplus B) \Theta B
$$

Where A is a set of images, B is the structural element

The basic idea of mathematical morphology is using a "structural element" needle to collect image information, and the structural element is a set of Euclidean space or its subspace with certain geometric shapes $[9,10]$. Structural element is a very important concept of morphology, which is the key to morphology operation. The choice of different structural 
elements will lead to different analysis and processon geometry information, which will decide data distribution and data volume in the morphological transform, and it determines the complexity of the calculation. When the structural element is moving constantly in the image, you can inspect interrelation in various parts of the image, to understand the structural features in the various parts of the image.

In this paper, according to the characteristics of the rectangular plate we are carrying on mathematical morphological operation,vertical texture will be merged to obtain the license macroscopic shape characteristic; Taking mathematical morphology and fuzzy evaluation function as the carrier, we analyze comprehensively plate texture structure and macro-structure information, it will enhance the accuracy and anti-jamming of the car license location. Point-based mathematical morphology operations will be extended to form line-based mathematical morphology algorithms.

Mathematical morphology operations for erosion and opening transform of objects are used for implementing thinning and pruning algorithms, which will be calculated to increase the accuracy of segmentation process.

\subsection{Thinning Algorithm}

A good thinning algorithm should preserve the connectivity and remove pixels from a shape in an image until the width of the skeleton to unity.

In this paper, a new structural element is presented for the morphological operations. At first the size of structural element is based on the size of the input image. And another major reason on designing structure element is based on the maximum interval between characters, which will try to ensure that all characters can form a single connected domain.Because binary edge image bySobel testing highlights the vertical texture,so we firstly carry on the inflation operation by using level structural elements; And in the experiment the distance between the edges of license plate area is usually 6 to 8 pixels, the distance between characters in image is often 3 to 4 pixels. Moreover, considering that some edges may be missed, the length of level structure elements is [10 1] through numbers of experiments. Because the width of structure element is slightly larger than or equal to the width of characters spacing, the license plate area will be filled with solid after inflation operation, generally the license plate area can achieve connection after a dilation operation and also inhibit other non-plate region to achieve connection. Opening operation with vertical structural elements selects [1 6], which is eliminated scattered points in the image. After the above processing, the car license region will be more obvious, as be shown in Figure 4.

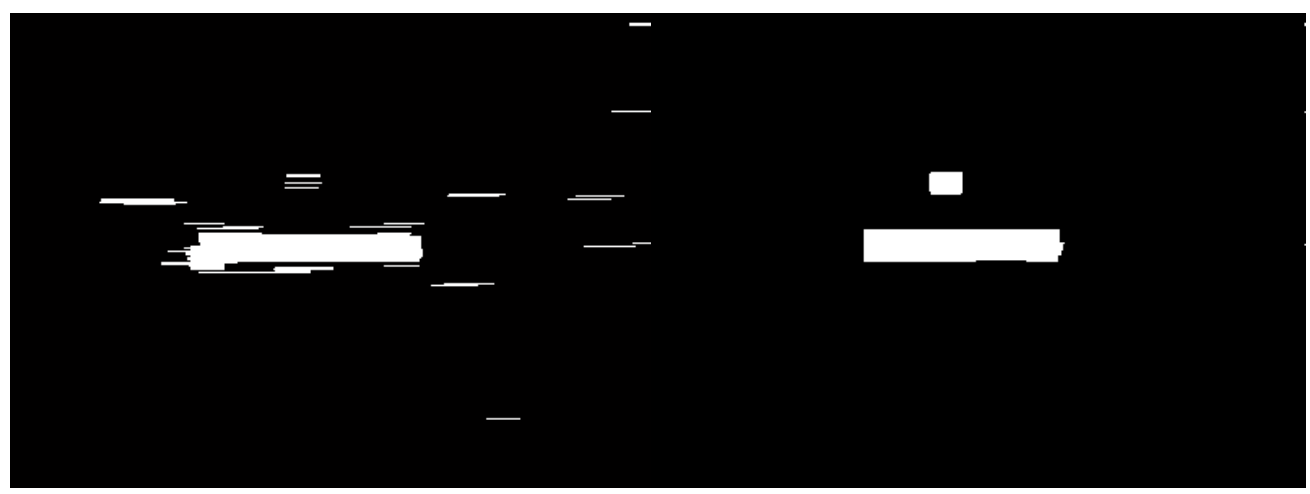




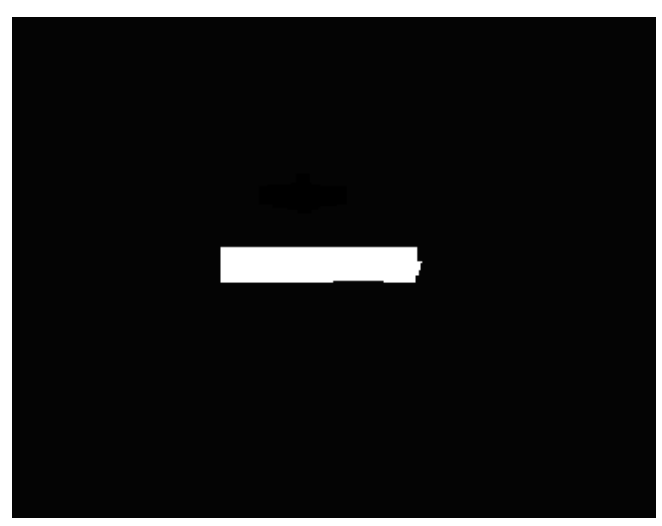

Figure 4. Processing Results of Morphology

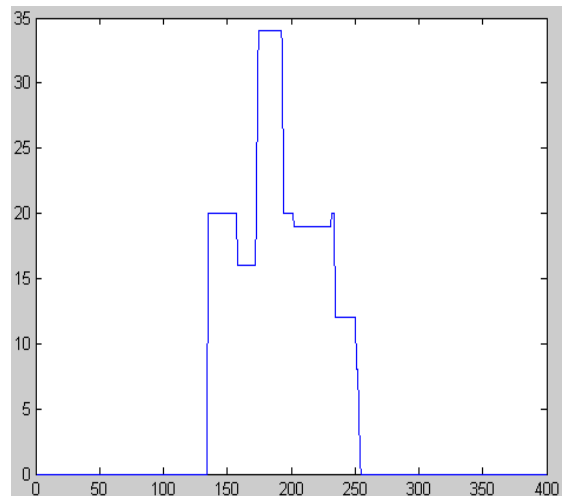

X-axis Projection Histogram

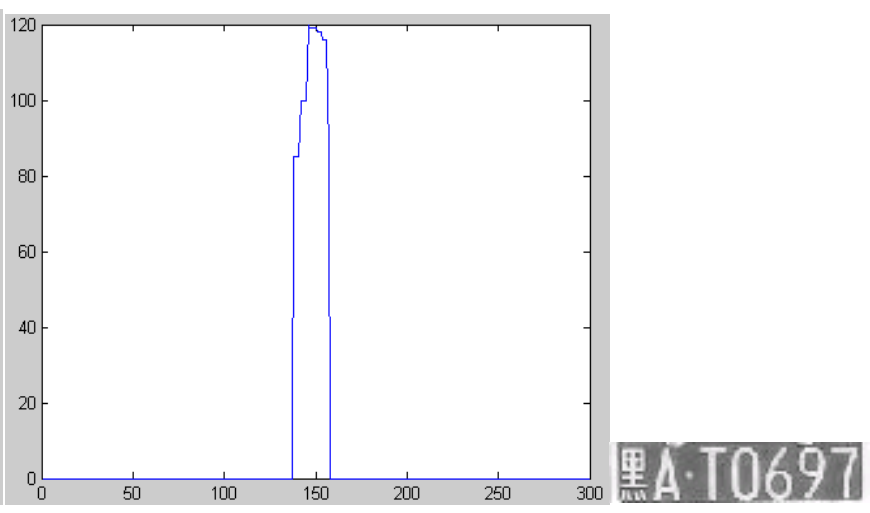

Y-axis Projection Histogramthe Plate Region

\subsection{Pruning Algorithm}

The pruning algorithm is an essential complement to the thinning algorithm. By the above morphological processing, many isolated connected regions are formed in the image, which can obtain several candidate areas, including the car license, the headlight, the trademark etc. By some single evaluating indicator,it is difficult to locate the license plate area from numerous candidate areas accurately. So in this paper we consider a variety of factors to determine the license plate area. According to the characteristics of the license plate in China, the criterions basing on the ratio of plate area, ratio of height to width and projections are used in comprehensive analysis, and the real license plate area can be located accurately.

Because the distance between CCD and the automobile is usually fixed in filming, so the taken image has fixed proportion size, that is the height, the width of the license plate region, the ratio of width to height, and area which should be in certain limits.

$$
R(i)=\frac{\text { Width }(i)}{H e i g h t(i)} \operatorname{Sr}(i)=\frac{S(i)}{S n}
$$

In these formulas, $\mathrm{R}(\mathrm{i})$ indicate the high-width ratio of the $\mathrm{i}$ candidate region, $\mathrm{Sr}(\mathrm{i})$ indicate the ratio of the $\mathrm{i}$ candidate area and the whole license plate image. According to the prior knowledge on the license plate, and the real candidate region is in the middle of the body area, so the width-height ratio $\mathrm{R}$ is approximately: $\mathrm{W} / \mathrm{H}=140 / 440=0.318$, in this paper we take [0.3-0.4] as judgment interval,Sr takes [0.01-0.04]. Accordingly most of the candidate regions can be deleted, as shown in figure 4. Finally, we can distinguish real license plate region by 
the vertical projection. And the plate is cut out from the original image. Because the license plate is an approximate rectangle or parallelogram area, so we need to carry on the division to a rectangular region. Segmentation method is mark connected region on license plate, on the marked boundary connected region we find out separately maximum and the minimum value of the line and the row vector, which will confirm the four endpoints of the rectangle, and according to its coordinates to be cut, toget license plate image.

\section{Experimental Result Analysis}

The proposed method was implemented on a personal computer with an Intel core(TM)2 CPU by Matlab 7.0. Inordertoanalyzetheperformanceofproposedapproach, 200images from CCDareusedfortesting. Thesizeofeachimageis $640 * 480$.To increase the complexity of the test database, images are acquired at different conditions including various backgrounds (Contains doubleplates), all kinds of size (cars, jeeps, vans, trucks etc), light conditions (cloudy and sunny, day and night) and contrast. The average accuracy of license plate detection is nearly $98 \%$. The superiority of the proposed method can be verified by the experimental results. Wrong localization the reason mainly to have the following two aspects: First, the car license mud and dust pollution is too serious. Second, other characters in image disturbance cause the background extremely complex. These will be further study and improve.

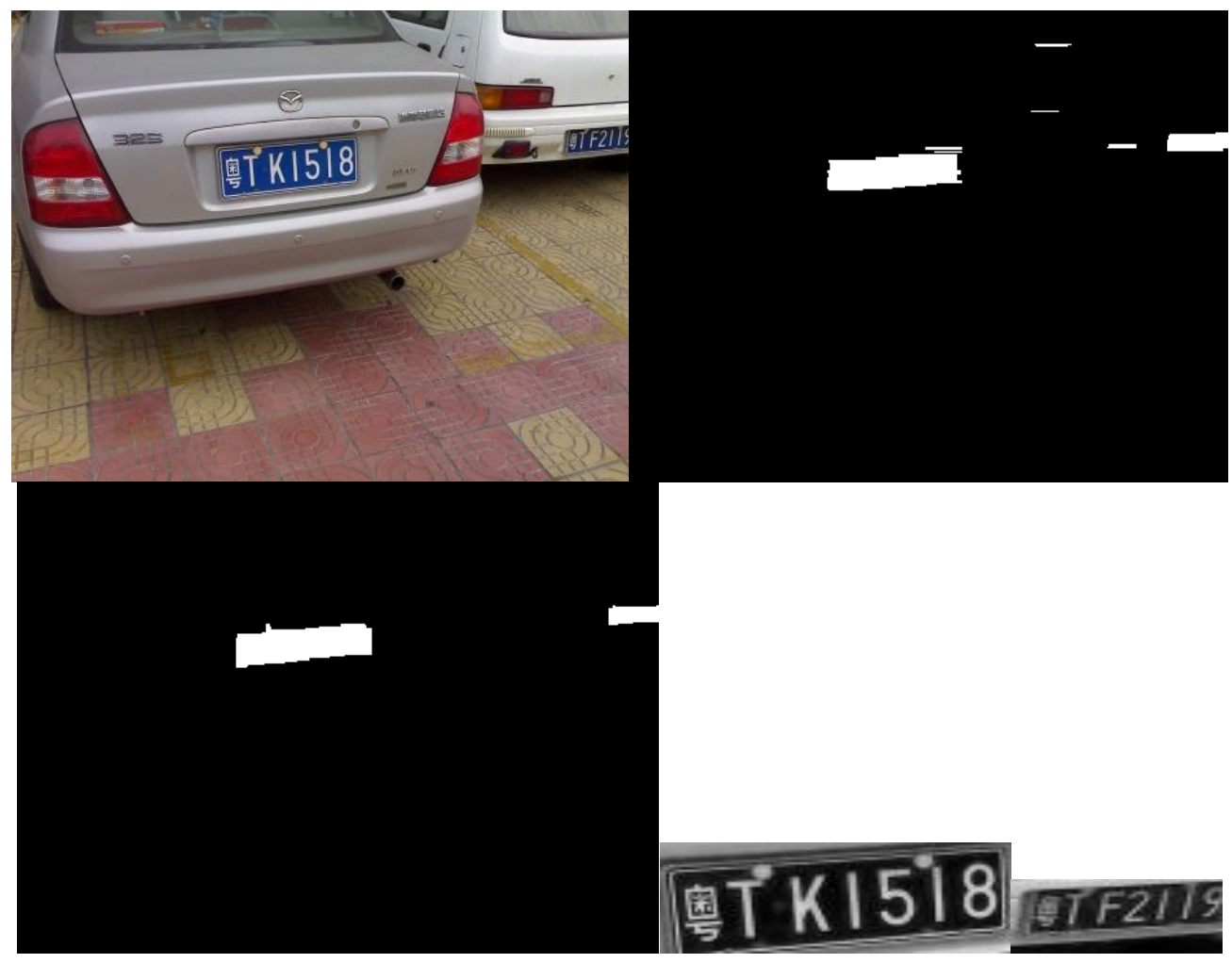

Figure 5. Double Plates Locating Experimental Result 


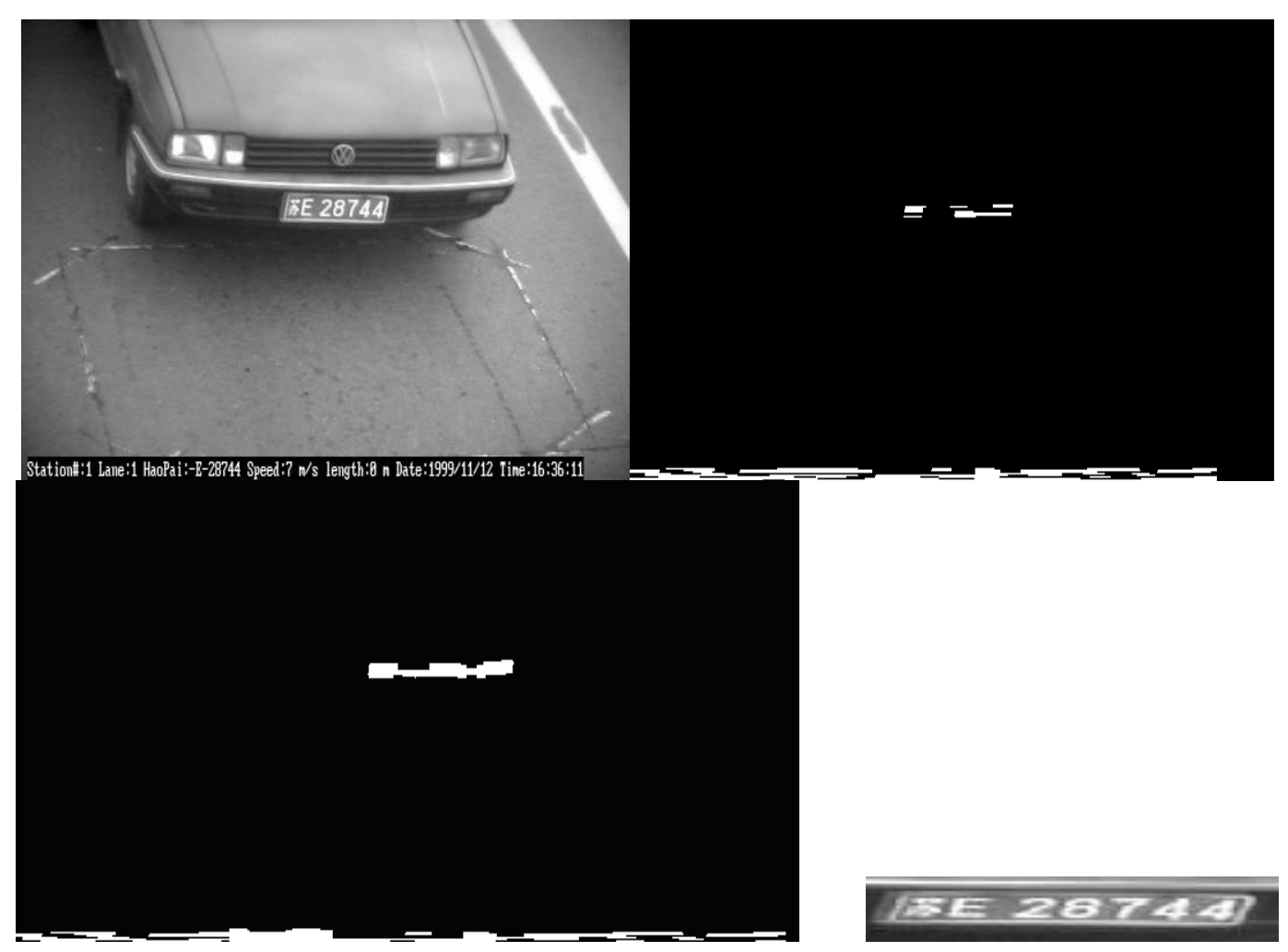

Figure 6. Plate Locating Experimental Results in Nighttime

\section{Conclusion}

A great effect of our method in license plate location has been confirmed by the experiments. In this paper the author presents a new method of license plate location, that gray-scale image using the improved Sobel operator for edge detection and mathematical morphology combination of license plate location method.The theoretical analysis and simulation have proved that the algorithm is effective. This algorithm is better than the general algorithms, which is able to remove the noise, the edge detail loss is small, the smooth effect is good, edge clear and continuous, and is easy to program implementation. And some of the ideas in this method can be applied to some other applications, such as "text extraction on brands, cards, bills ...", "object segmentation in complex background" etc.

\section{Acknowledgements}

This work was supported by Science and Technology Research Projects of Heilongjiang Education Committee (12531190).

\section{References}

[1] S. Singh,Chhabada, R. Singh, and A.Negi, "Heuristics For License Plate Detection And Extraction", World Journal of Science and Technology, vol. 1, no.12, (2011), pp. 63-67.

[2] Y.Q. Lu andL.C. Wu, "A Method of License Plate Location Based on Morphology",Computer Engineering,vol. 31, (2005),pp. 224-225.

[3] W. AiliamdX. Liu, "Vehicle License Plate Location Based on Improved Roberts Operator and Mathematical Morphology",Instrumentation Measurement Computer Communication and Control (IMCCC),2012Second International Conference on.IEEE, (2012).

[4] G. Li,R.L. Zeng,L. Lin,M.J.Wang, "Car license plate location algorithm based on mathematical 
morphology",Chinese Journal of Scientific Instrument,vol. 28, no. 7, (2007),pp. 1323-1327

[5] X.C.Jiang,Z.K. Wan,L. Chen, "The Comparison of Several Edge Detection Methods Based on Matlab", Computer Knowledge and Technology,vol. 1, (2006), pp. 138-141.

[6] J. Wenjing, H. Zhang, X. He; M. Piccardi, "License Plate Localization from Vehicle Images An Edge Based Multi-stage Approach”, In IEEE Intelligent Transportation Systems, (2009),pp. 566-571.

[7] Y.L. Ma andQ.H. Xia, "Algorithmic Research of License Plate Location Based on Mathematics Morphology",Control \& Automation,vol. 24, no. 1, (2008), pp.227-228.

[8] W. Pan and R.An, "Morphology and auto-correlation based method of fast locating vehicle license plate", The 2nd International Conference on Advanced Computer Control (ICACC), vol. 3, (2010), pp.116-119.

[9] L.Zhou, Y. Liu, “An Approach Based on Morphology for Car License Plate Extraction”, Journal of Qingdao University of Science and Technology,vol. 28, no. 1, (2007), pp.69-72.

[10] B.-W. Zhang, X.-L.Tian, Y.-K Sun,"Based on the Improved Mathematical Morphology OCT Image Quick Edge Detection Algorithm”, ComputerScience, vol. 6, (2013), pp. 173-175

\section{Authors}

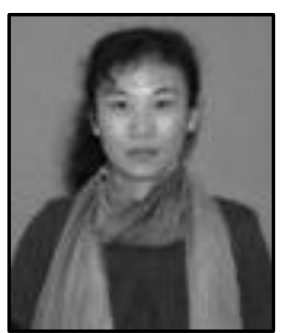

Yanling Cui, she received her M.Sc. in computer application (2009) from HarbinNormalUniversity. Now she is a lecturer of Institute of Computer Science and Information Engineering. Her current research interests include different aspects of image processing and pattern recognition.

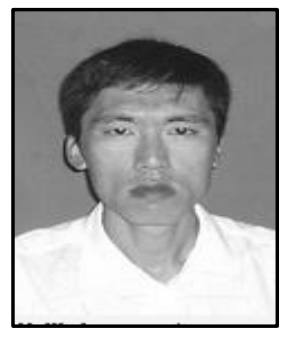

Chengjun Yang, he received his M.Agr. in Agronomy from YanbianUniversity (2005) and $\mathrm{PhD}$ in Agronomy(2008) from NortheastForestryUniversity. Now he is associateprofessor of forestry at Forest Plant Resources Department.His current research interest is bioinformatics. 
International Journal of Control and Automation Vol.7, No.9 (2014) 